\title{
Esophageal 24-Hour pH-Metry after Esophageal Manometry Facilitated by a New Medical Device, A Mini-Overtube
}

\author{
Benedetto Mangiavillano*,1, Sandro Passaretti ${ }^{2}$, Maura Corsetti ${ }^{2}$, Gianni Mezzi ${ }^{2}$, Simona Curioni ${ }^{2}$ \\ and Pier Alberto Testoni ${ }^{2}$
}

\begin{abstract}
${ }^{1}$ Department of Gastrointestinal Endoscopy, University San Paolo Hospital; ${ }^{2}$ Physiopathology Digestive Laboratory, Gastroenterology and Gastrointestinal Endoscopy Unit, Vita-Salute San Raffaele University, Scientific Institute San Raffaele, Milan, Italy
\end{abstract}

\begin{abstract}
Esophageal manometry (EM) and ambulatory 24-hour esophageal pH-metry (EP) are techniques employed in the management of patients with gastroesophageal reflux disease (GERD). For these examinations, two consecutive probes must be placed nasally. To evaluate the introduction-time (IT) and patient tolerance (PT) during introduction of the EP probe after EM with and without the assistance of a new medical device, a mini-overtube (MOT). A series of 115 patients referred to our Gastroenterology Unit to undergo EM and EP were divided into two groups: group 1 underwent EM and EP by the traditional procedure, group 2 with the MOT. The mean IT for the EP probe was significantly shorter in group 2 than group $1(7.4 \pm 2.0 \mathrm{sec}$ vs. $93.2 \pm 57.1 \mathrm{sec} ; \mathrm{p}<0.01)$. In group 1 no patients reported excellent tolerance to introduction of the EP probe, $22.7 \%$ reported good tolerance, $50 \%$ medium and $29.3 \%$ bad. In group 2, $61.4 \%$ reported excellent tolerance, $33.3 \%$ good, 5.3\% medium and none bad. Six group 1 patients $(10.3 \%)$ refused the introduction of the EP probe after $239.7 \pm 113.9$ seconds of unsuccessful efforts. This device facilitates the introduction of the EP probe in all patients, even those who would not otherwise agree to a second attempt.
\end{abstract}

\section{INTRODUCTION}

Esophageal manometry (EM) and 24-hour esophageal pH-metry (EP) are accepted clinical tools for investigating esophageal disease [1,2]. These procedures are most frequently required in patients with dysphagia but no evidence of esophageal mechanical obstruction, and in patients with suspected non-cardiac chest pain or gastro-esophageal reflux disease (GERD). GERD is in fact the most common indication for EM and EP [3]. The standard approach in most esophageal motility units uses a water-perfused multichannel catheter system connected indirectly to a physiograph through a series of transducers [4-6]. EM was primarily employed in the past to check low esophageal sphincter (LES) pressure but it is not indicated for placement of a $\mathrm{pH}$ probe. Manometry is in fact recommended for precise positioning of the $\mathrm{pH}$ probe $5 \mathrm{~cm}$ above the upper margin of the LES $[7,8]$.

Prolonged ambulatory monitoring of esophageal $\mathrm{pH}-$ metry is the most reliable method for diagnosing GERD. First described by Spencer in 1969 and popularized by Johnson and DeMeester in 1974 [9], the procedure evolved from dissatisfaction with previous tests for reflux. Data are collected for 24 hours in a small lightweight box worn on a waist belt and the information is analyzed by computer. $\mathrm{Pa}$ tients are allowed to smoke and drink alcohol and most centers no longer restrict the $\mathrm{pH}$ of foods.

*Address correspondence to this author at the Dept. of Gastrointestinal Endoscopy, San Paolo University Hospital, Via A. di Rudinì 8, 20142 Milan, Italy; Tel: 003902 8184-4273-4057; Fax: 003902 89125264; E-mail: b_mangiavillano@hotmail.com
However, each time patients need both EM and EP, two separate probes have to be introduced. Generally introduction of the EM probe is comfortable enough because of the blunt distal end but the EP probe is not nearly so well tolerated. Nasal intubation is complicated by its excessive flexibility and the distal end, made of glass or antimony, can cause trauma to the nasal mucosa, small bleeds, repeated sneezing, pain in the nostril, and tears. Even when the $\mathrm{pH}$ probe is introduced, it often takes much longer than the EM probe. Many patients insist on stopping attempts at introducing $\mathrm{pH}$-metry or $\mathrm{pH}$-impedance probes because of the discomfort or pain [10].

To overcome this problem we tested a new medical device, a mini-overtube (MOT), in patients requiring EM with EP. We also evaluated intubation time (IT) and patient tolerance (PT) with and without the MOT.

\section{MATERIALS AND METHODS}

We consecutively enrolled 115 patients referred to our center for EM with EP and divided them into two groups: group 1 comprised 58 patients $(33 \mathrm{~F}$ and $25 \mathrm{M}$; mean age $52.5 \pm 15.3$ years) who were examined by EM and EP with the traditional technique; group 2 comprised 57 patients (29 $\mathrm{F}$ and $28 \mathrm{M}$; mean age $52.6 \pm 15.4$ years), in whom the examinations were "facilitated" with the MOT. All patients gave their written informed consent to the EM and EP, with or without the MOT, and to the publication of their data for scientific purposes.

The MOT is a small tube made of polyethylene, a material that allows the esophageal manometric probe to skim through without any resistance. The new device is $30 \mathrm{~cm}$ 


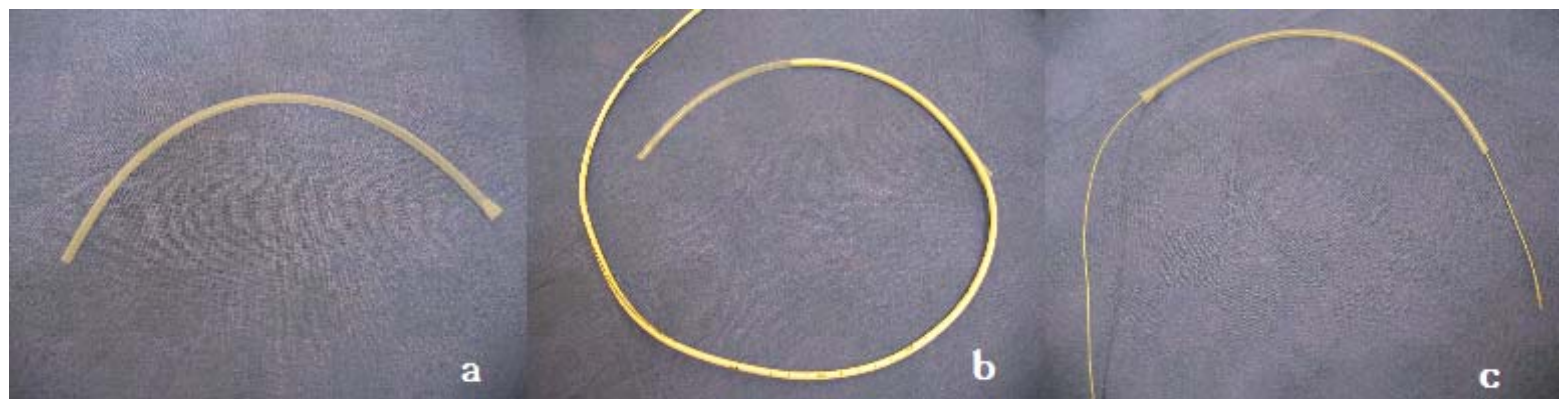

Fig. (1). (a) MOT with the funnel-shaped distal extremity to facilitate introduction of the probes. (b) The MOT put over the manometric probe. (c) The EP probe inserted into the MOT.

Table 1. Intubation Time (IT) in the Two Groups

\begin{tabular}{|c|c|c|c|}
\hline & $\begin{array}{c}\text { Group 1 } \\
(\mathbf{n = 5 7} \mathbf{~ p t s})\end{array}$ & $\begin{array}{c}\text { Group 2 } \\
(\mathbf{n}=\mathbf{5 8} \text { pts })\end{array}$ & $\mathbf{p}$ \\
\hline \hline Intubation Time (IT) (in seconds) & $93.2 \pm 57.1$ & $7.4 \pm 2.0$ & $\mathrm{p}<0.01$ \\
\hline
\end{tabular}

Table 2. Patients' Tolerance (PT) to pH-Probe Introduction in the Two Groups

\begin{tabular}{|c|c|c|c|}
\hline Tolerance to pH Probe Introduction & Group 1 & Group 2 & p \\
\hline \hline Excellent & $0 \%(0 \mathrm{pts})$ & $61.4 \%(35 \mathrm{pts})$ & $<0.01$ \\
\hline Good & $22.7 \%(12 \mathrm{pts})$ & $33.3 \%(19 \mathrm{pts})$ & $\mathrm{ns}$ \\
\hline Medium & $50 \%(29 \mathrm{pts})$ & $0.3 \%(3 \mathrm{pts})$ & $<0.01$ \\
\hline Bad & $29.3 \%(17 \mathrm{pts})$ & $0 \%(0 \mathrm{pts})$ & $<0.01$ \\
\hline
\end{tabular}

long, curved, with a 20 -cm cord, 13 Fr inner diameter and 14.5 Fr outer diameter. Its distal extremity is funnel-shaped, to facilitate introduction of the $\mathrm{pH}$-metry probe. The proximal part of the MOT is blunted to avoid trauma during transnasal introduction (Bioengineering Laboratories s.p.a., Cantù, CO, Italy).

Before starting EM, the MOT is placed over the manometric probe at its distal extremity. EM is done using a six-lumen, water-perfused, PVC esophageal manometry catheter, with maximum diameter $4 \mathrm{~mm}$ (12 Fr) (Bioengineering Laboratories s.p.a., Cantù, CO, Italy). After EM, the MOT is skimmed down over the manometric probe, before it is extracted, so that the distal extremity goes some centimeters beyond the upper esophageal sphincter (UES). Then, when the EM probe is extracted, with the MOT still stationary over the UES, the EP probe is inserted inside the MOT, which serves as a guide. Subsequently the MOT is pulled back on the EP probe, up to the electrical connector, and left on the probe throughout the examination (Fig. 1).

For 24-h EP monitoring we used a glass electrode (diameter $1.5 \mathrm{~mm}$ ) connected to a portable digital memory unit (LoT 142; Mettler Toledo, M.I.C., Champagne au Mont D'Or; France). We defined the EP probe IT as the time needed to insert the probe into the esophagus, starting when the distal tip is at the nostril and stopping when the proximal marker is at the nostril. The marker is put on the probe after EM when the distance from the nostril to the LES has been obtained. We also assessed patients' tolerance (PT) to intro- duction of the $\mathrm{pH}$ probe, rating it on a subjective scale as bad, medium, good or excellent.

\section{STATISTICAL ANALYSIS}

As the data showed a Gaussian distribution, Student's ttest for unpaired data was used, and Fisher's exact test for the frequency data. Data are presented as median \pm standard deviation.

\section{RESULTS}

The EP probe IT was 12.6 times shorter in group 2, where the procedure was facilitated with the MOT, than group 1 (93.2 \pm 57.1 vs. $7.4 \pm 2.0 ;$ p<0.01) (Table 1). Six patients $(10.3 \%)$ in group 1 refused further attempts at introducing the EP probe after $239.7 \pm 113.9 \mathrm{sec}$ of unsuccessful tries.

In group 1 no patients reported excellent tolerance to introduction of the probe, 12 rated it good (22.7\%), 29 medium $(50 \%)$ and 17 bad $(29.3 \%)$. In group 2, 35 patients reported excellent tolerance $(61.4 \%), 19$ good $(33.3 \%), 3$ medium $(5.3 \%)$ and none bad. The difference was significant $(\mathrm{p}<0.01)$ except for good tolerance (Table 2).

\section{DISCUSSION}

Esophageal manometry has to be done to place a $\mathrm{pH}$ metry probe correctly. With this technique the distance from the nostril to the LES can be measured, though Showalter et 
al. reported a mean difference of up to $0.7 \mathrm{~cm}$ in the radially determined location of the lower esophageal margin [11]. EM is currently employed in many centers, but it is followed by EP only in highly specialized referral units. Patients tolerate the manometry well, but some of them refuse EP because of intolerance to the introduction of the $\mathrm{pH}$ probe, or consequences such as small hemorrhages, repeated sneezing, pain in a nostril, or tears, probably caused by the potentially traumatic distal tip of the probe. It is always easier and less traumatic to introduce the manometry probe than the $\mathrm{pH}$ probe, and patients tolerate it better. This is because of the material it is made of and the non-traumatic distal tip.

The new device avoids the risk of trauma from the probe when pH-metry is done after EM. It also allows a single intubation and reduces the IT by 12.6 times compared to the traditional procedure, giving a better quality examination.

Finally, besides remarkably easing the introduction of the EP probe, the MOT enables the probe to be introduced in all patients, even those who would normally not agree to a second attempt. In our opinion this new device should be used for every patient needing both EM and EP, considering its utility and ease of use.

\section{CONCLUSION}

With the MOT the EP probe can be introduced, with significant time saving, in all patients, even those who would not otherwise agree to a second probe introduction.

\section{REFERENCES}

[1] Castell DO. Historical perspective and current use of esophageal manometry. Esophageal motility testing. Amsterdam: Elsevier 1987.

[2] Castell DO, Wu WC, Ott DJ. Gastroesophageal reflux disease, pathogenesis, diagnosis, therapy. New York: Futura 1985.

[3] Steff JJ, Dodds WJ, Hogan WJ. Intraluminal esophageal manometry: an analysis of variables affecting recording fidelity of peristaltic pressures. Gastroenterology 1974; 67: 221.

[4] Dodds WJ. Instrumentation and methods for intraluminal esophageal manometry. Arch Intern Med 1976; 126: 515.

[5] Janssens J, Vantrappen G, Ghillebert G. 24-hour recording of esophageal pressure and $\mathrm{pH}$ in patients with non-cardiac chest pain. Gastroenterology 1986; 90: 1978-4.

[6] Malagelada JR, Stanghellini V. Manometric evaluation of functional upper gut symptoms. Gastroenterology 1985; 88: 1223-31.

[7] Kahrilas PJ, Quigley EMM. American Gastroenterological Association medical position statement: guidelines in the use of esophageal pH recording. Gastroenterology 1996; 110: 1981-96.

[8] DeVault KR, Castell DO. Up-to-date guidelines for the diagnosis and treatment of gastroesophageal reflux disease. Am J Gastroenterol 1999; 94: 1434-42.

[9] Johnson LF, DeMeesyer TR. Twenty-four-hour $\mathrm{pH}$ monitoring of the distal esophagus: a quantitative measure of gastroesophageal reflux. Am J Gastroenterol 1974; 62: 325-32.

[10] Richter JE, Bradley LA, DeMeester TR, Wu WC. Normal 24-hr ambulatory esophageal $\mathrm{pH}$ values. Influence of study center, $\mathrm{pH}$ electrode, age and gender. Dig Dis Sci 1992; 37: 849-56.

[11] Kaye MD, Showalter JP. Manometric configuration of the lower esophageal sphincter in normal human subjects. Gastroenterology 1971; 61: 213-23.

Received: May 29, 2008

() Mangiavillano et al.; Licensee Bentham Open.

This is an open access article licensed under the terms of the Creative Commons Attribution Non-Commercial License (http://creativecommons.org/licenses/by-nc/3.0/) which permits unrestricted, non-commercial use, distribution and reproduction in any medium, provided the work is properly cited. 